\title{
Prevalência dos Subtipos Histológicos do Câncer de Mama em Pacientes atendidas em um Serviço de Oncologia de Alta Complexidade e Demanda do sul de Santa Catarina de 2006 a 2014.
}

\author{
Ana Paula Lazaretti'; Priscila Marques de Assis ${ }^{1}$; Livia Machado \\ Scridelli ${ }^{1}$; Juliana Lorenzoni Alhtoff ${ }^{2}$, Kleber Serafim Daltoé3; \\ Kristian Madeira ${ }^{4}$. \\ 1.Graduandas em Medicina da Universidade do Extremo Sul Catarinense (UNESC), Criciúma -SC. \\ 2.Médica. Especialista em Cancerologia - Hospital de Clínicas de Porto Alegre. Mestre em Medicina e \\ Ciências da Saúde - Pontifica Universidade Católica de Porto Alegre, Professora do curso de Medicina - \\ UNESC. \\ 3.Médico. Especialista em Cancerologia - Hospital Governador Celso Ramos. Professor do curso de \\ Medicina - UNESC \\ 4.Professor de Bioestatística do curso de Medicina - UNESC. Doutor em Ciências da Saúde pela UNESC.
}

\section{Resumo}

Introdução: $O$ câncer de mama é a neoplasia maligna mais comum em mulheres. No Brasil, segundo dados do Instituto Nacional do Câncer (INCA), excluindo os tumores de pele não melanoma, é o câncer feminino mais prevalente em quase todas as regiões do país, com exceção da região norte, onde este tumor ocupa o segundo lugar. São responsáveis por aproximadamente $12 \%$ de todos os novos casos de câncer e $25 \%$ do total de cânceres no sexo feminino. Dentre os subtipos histológicos, o carcinoma ductal invasor (CDI) é o tipo mais comum de câncer de mama invasivo, correspondendo a 70 a $80 \%$ dos casos, as características citológicas variam de brandas à altamente malignas, essas células induzem uma resposta fibrosa à medida que se infiltram no parênquima mamário, e esta reação é, em grande parte, responsável pela massa palpável detectável clinicamente, além da densidade radiológica e ecográfica típicas de carcinomas 
invasivos. Em segundo lugar encontra-se o carcinoma lobular invasivo (CLI), que abrange 5 a $10 \%$ das lesões invasivas, alguns carcinomas lobulares infiltrantes apresentam aspecto macroscópico idêntico ao de carcinoma ductal infiltrante, em muitos casos, sem lesão de massa evidente, e o tecido mamário retirado pode ter uma consistência normal ou apenas ligeiramente firme. Assim, o tamanho microscópico do carcinoma lobular invasivo pode ser significativamente maior do que o esperado. $\mathrm{O}$ carcinoma mucinoso representa entre 1 a $2 \%$ dos casos de câncer de mama invasivo e é mais comum em pacientes idosas, e quanto maior a quantidade de mucina, melhor o prognóstico. Nos exames de imagem, o carcinoma mucinoso se apresenta como um nódulo de formato ovóide ou redondo, com margens circunscritas, podendo ser confundido com uma lesão benigna, retardando o diagnóstico e tratamento correto. O carcinoma medular é responsável por 1 a 10\% dos casos de câncer de mama invasivo, ocorrendo principalmente em mulheres jovens (menores de 35 anos). Assim como o carcinoma mucinoso, o medular é clinicamente, um tumor bem delimitado, e na mamografia é tipicamente bem circunscrito, facilmente confundido com fibroadenoma. As células tumorais são pouco diferenciadas, todavia, esse subtipo apresenta prognóstico relativamente favorável. Também existem subtipos mais raros, aonde se enquadram Carcinoma papilar, Carcinoma cribiforme, Carcinoma tubular e Carcinoma pouco diferenciado. Objetivo: Avaliar a prevalência dos subtipos histológicos no Câncer de Mama em pacientes diagnosticadas com doença invasora em um Serviço de Oncologia de Alta Complexidade e Alta Demanda do Sul do Estado de Santa Catarina, no período de 2006 a 2014. Metodologia: Foi realizado um estudo transversal, documental e retrospectivo, avaliando prontuários de pacientes do sexo feminino com 
diagnóstico de câncer de mama invasor, atendidas em um Serviço de Oncologia de Alta Complexidade e Alta Demanda do Sul do Estado de Santa Catarina, no período de 2006 a 2014. Os critérios de exclusão foram prontuários incompletos ou não localizados e pacientes com metástases à distância ao diagnóstico. Os dados coletados foram organizados em planilhas do software Statistical Package for the Social Sciencies (SPSS) versão 22.0, os quais foram analisados em frequência e porcentagem. Resultados: Foram analisados 387 prontuários. 327 (84,5\%) das pacientes apresentaram Carcinoma Ductal Invasor (CDI), 45 (11,6\%) apresentaram Carcinoma Lobular Invasor, 6 (1,6\%) apresentaram Carcinoma Mucinoso, $4(1,0 \%)$ apresentaram Carcinoma Medular e $9(2,3 \%)$ se enquadraram entre os outros subtipos, incluindo nesse grupo: Carcinoma papilar, Carcinoma Cribiforme, Carcinoma Tubular e Carcinoma Pouco Diferenciado. Conclusão: De acordo com os dados analisados conclui-se que o subtipo mais prevalente é o CDI, seguido pelo CLI, e com poucos casos de subtipos histológicos mais raros o que corrobora com a literatura atual que apresenta estimativas semelhantes às encontradas no presente estudo.

Palavras Chave: Câncer de Mama; Subtipos Histológicos; Mastologia 


\section{Referências}

BLEIWEIISS, I.J. Pathology of breast cancer [online] Disponivel em Up To Date. Arquivo acessado em 14 de janeiro de 2015.

COELLI, G.N.M. et al. Carcinoma mucinoso da mama: ensaio iconográfico com correlação histopatológica. Rev. Radiol Bras. v.46, n.4, p. 242-246, 2013.

INCA (Instituto Nacional do Câncer). Mama. [online] Disponível na Internet via WWW.

URL:

http://www2.inca.gov.br/wps/wcm/connect/tiposdecancer/site/home/mama. Acessado em 15 de janeiro de 2015.

MATHEUS, V. L. et al. Carcinoma medular da mama: correlação anátomo-radiológica. Rev. Radiol Bras. v.41, n.6, p.379-383, 2008.

WORLD CANCER RESEARCH FUND INTERNATIONAL. Breast cancer statistics. [online] Disponível na Internet via WWW. URL: http://www.wcrf.org/int/cancer-factsfigures/data-specific-cancers/breast-cancer-statistics. Acessado em 04 de dezembro de 2014. 